TITLE:

\title{
A rewriting system for categorical combinators with multiple arguments
}

$\operatorname{AUTHOR}(\mathrm{S})$ :

Yokouchi, Hirofumi; Hikita, Teruo

\section{CITATION:}

Yokouchi, Hirofumi ... [et al]. A rewriting system for categorical combinators with multiple arguments. 数理解析研究所講究録 1988, 655: 186-208

ISSUE DATE:

1988-04

URL:

http://hdl.handle.net/2433/100507

RIGHT: 


\section{A rewriting system for categorical combinators with multiple arguments}

Hirofumi Yokouchi

粗内寡文

Tokyo Research Laboratory, IBM Japan

Chiyoda, Tokyo 102, Japan

Teruo Hikita

\section{正田枓椎}

Department of Mathematics, Tokyo Metropolitan University

Setagaya, Tokyo 158, Japan

Version: 26 October 1987

Abstract. Categorical combinators have been derived from the study of categorical semantics of lambda calculus, and it has been found that they may be used in implementation of functional languages. In this paper categorical combinators are extended so that functions with multiple arguments can be directly handled, thus making them more suitable for practical computation. A rewriting system named $C C L M_{\beta}$ is formulated for these combinators. In this system partial computation is naturally realized. The relationship between this system and lambda calculus is established. As a result of this, the Church-Rosser property of the system is proved.

Key words. Categorical combinator, Church-Rosser property, combinator, functional programming, lambda calculus, partial computation, rewriting system 


\section{Introduction.}

Categorical models of lambda calculus have been extensively studied, e.g. [2], [7], [8], [9], [10], [11], [13]. Curien [4], [5] introduced categorical combinators from such categorical semantics of lambda calculus, and he formulated rewriting systems for them, such as $C C L_{\beta}$ and $C C L_{\beta \eta S P}$. Yokouchi [14] independently introduced the CCM calculus, which is equationally equivalent to $C C L_{\beta}$ but has slightly different rewriting rules. Incidentally, these systems have a strong resemblance to the functional style language FP of Backus [1]. Categorical combinators have been used in implementation of functional languages [3].

Partial computation, or often called partial evaluation, is a method of computing a function with more than one argument by supplying values to only a specified part of the arguments [6]. It has many applications such as compiler generation.

All the functions associated with the categorical combinators treated so far are presumably one-argument. But this is not directly suited for practical computation where multipleargument functions are prevailing. Intended for practical application, in this paper a new extended set of categorical combinators is introduced in order to incorporate the notion of functions with multiple arguments. The starting point of the idea is to introduce $n$-tuples for arbitrary $n \geq 0$, instead of only pairs in the previous categorical combinators. Related to this, we assume that every function in the system has its own fixed arity, the number of arguments. With these extensions, the operations of currying and application are naturally extended to "partial currying" and "partial application".

A rewriting system named $C C L M_{\beta}$ is formulated for these new categorical combinators. One restriction of this system is that $n$-tuples are not allowed to appear in themselves: they may not appear at "top level", so that tuple-valued functions are not treated. Partial computation is naturally realized in this system. The system is semantically equivalent to type-free $\lambda_{\beta}$-calculus (without product). Moreover, our results show that these two systems are equivalent even in the sense of reduction. We will establish the natural relationship between the system $C C L M_{\beta}$ and the lambda calculus by giving translation algorithms between the two. The Church-Rosser property of the system, which is not obtained for the systems $C C L_{\beta}$ and $C C L_{\beta \eta S P}$, is proved through this relationship with lambda calculus.

In Section 2 the new categorical combinators are introduced, and the rewriting system $C C L M_{\beta}$ is formulated for these combinators. A simple example of computation in this system is also given. In Section 3 we briefly state the model theoretic aspect of the system. In Section 4 some derived combinators are introduced which will be useful in practical 
computation. In Section 5 the translation algorithms are introduced between the $C C L M_{\beta}$ calculus and lambda calculus, and in Section 6 theorems on the relationship concerning reduction between these two systems are established. Finally, the Church-Rosser property of $C C L M_{\beta}$ is proved in Section 7, using the results in Section 6.

We assume the reader the basic knowledge of lambda calculus (e.g. [2]). The acquaintance with categorical combinators $([4],[5])$ is desirable, but this paper is self-contained and makes no use of previous results about them. We are here mainly interested in the formal aspect of the system, so that application of the categorical combinators with multiple arguments to implementation of functional languages is left to a separate paper. 


\section{Rewriting system $C C L M_{\beta}$.}

Before presenting the formal system $C C L M_{\beta}$ of categorical combinators with multiple arguments, we explain the intuitive meaning of each of the new combinators. The combinator o means function composition. For $n$ functions $f_{1}, f_{2}, \ldots, f_{n},(n \geq 0)$, the angular brackets $\left\langle f_{1}, f_{2}, \ldots, f_{n}\right\rangle$ constitute an $n$-tuple. The combinator $p_{i}^{n}, 1 \leq i \leq n$, is the $i$-th projection of an $n$-tuple.

We extend the usual currying operation to functions with $n$ arguments. For $n \geq 1$, the combinator $\Lambda_{n}$ applies to a function with $n$ arguments and means currying. More precisely, for an $n$-argument function $f, \Lambda_{n}(f)$ is an $(n-1)$-argument function whose arguments correspond to the first $n-1$ arguments of $f$. The resulting value of the function $\Lambda_{n}(f)$ is a one-argument function, whose argument corresponds to the last argument of $f$.

We also extend the usual combinator of function application App to partial application. In our definition $A p p$ receives two arguments, an $n$-argument function and a value; it implies applying the value as the first argument among $n$ arguments of the function, and returns a function with $n-1$ arguments.

Now we formally give the definition of terms of $C C L M_{\beta}$.

Definition. We define terms with nonnegative integers called arity. In the following, terms are denoted by capital letters $F, G, F_{1}$, etc. For every constant its arity is uniquely specified. We assume that there are special constants: $p_{i}^{n}$ of arity $n$ where $n \geq 1$ and $1 \leq i \leq n$, and $A p p$ of arity 2 . Then the terms are defined inductively as follows.

(1) every constant is a term.

(2) if $F$ is a term of arity $m$ and $G_{1}, \ldots, G_{m}$ are terms of arity $n, m \geq 0, n \geq 0$, then $F \circ\left\langle G_{1}, \ldots, G_{m}\right\rangle^{n}$ is a term of arity $n$.

(3) if $F$ is a term of arity $n, n \geq 1$, then $\Lambda_{n}(F)$ is a term of arity $n-1$.

We omit the superscript $n$ of $F \circ\left\langle G_{1}, \ldots, G_{m}\right\rangle^{n}$ whenever no confusion occurs. Also, we often omit the subscript of $\Lambda_{n}(F)$ like $\Lambda(F)$.

Note that when $F$ is a term of arity $0, F \circ\langle\rangle^{n}$ is a term of arity $n$. Note also that in this system $n$-tuples in themselves are not terms; they always appear as part of composed 
terms.

Now we present the rewriting rules of the formal system $C C L M_{\beta}$.

Definition. We define the binary relation $\rightarrow$ among the terms of $C C L M_{\beta}$ by the following rules:

1. $\left(F \circ\left\langle G_{1}, \ldots, G_{m}\right\rangle^{n}\right) \circ\left\langle H_{1}, \ldots, H_{n}\right\rangle^{k} \rightarrow F \circ\left\langle G_{1} \circ\left\langle H_{1}, \ldots, H_{n}\right\rangle^{k}, \ldots, G_{m} \circ\left\langle H_{1}, \ldots, H_{n}\right\rangle^{k}\right\rangle^{k}$.

2. $p_{i}^{n} \circ\left\langle F_{1}, \ldots, F_{n}\right\rangle \rightarrow F_{i}$.

3. $F \circ\left\langle p_{1}^{n}, \ldots, p_{n}^{n}\right\rangle \rightarrow F$ where $F$ is of arity $n$.

4. $\Lambda_{n+1}(F) \circ\left\langle G_{1}, \ldots, G_{n}\right\rangle^{k}$ $\rightarrow \Lambda_{k+1}\left(F \circ\left\langle G_{1} \circ\left\langle p_{1}^{k+1}, \ldots, p_{k}^{k+1}\right\rangle, \ldots, G_{n} \circ\left\langle p_{1}^{k+1}, \ldots, p_{k}^{k+1}\right\rangle, p_{k+1}^{k+1}\right\rangle^{k+1}\right)$, where $F$ is of arity $(n+1)$, and $G_{1}, \ldots, G_{n}$ are of arity $k$.

5. $A p p \circ\left\langle\Lambda_{n+1}(F), G\right\rangle^{n} \rightarrow F \circ\left\langle p_{1}^{n}, \ldots, p_{n}^{n}, G\right\rangle^{n}$, where $F$ is of arity $n+1$, and $G$ is of arity $n$.

6. If $F \rightarrow F^{\prime}$, then $F \circ\left\langle G_{1}, \ldots, G_{m}\right\rangle \rightarrow F^{\prime} \circ\left\langle G_{1}, \ldots, G_{m}\right\rangle$.

7. If $G_{i} \rightarrow G_{i}^{\prime}$ for some $1 \leq i \leq m$, then $F \circ\left\langle G_{1}, \ldots, G_{i}, \ldots, G_{m}\right\rangle$

$\rightarrow F \circ\left\langle G_{1}, \ldots, G_{i}^{\prime}, \ldots, G_{m}\right\rangle$.

8. If $F \rightarrow F^{\prime}$, then $\Lambda(F) \rightarrow \Lambda\left(F^{\prime}\right)$.

We sometimes denote this relation $\rightarrow$ by $\rightarrow_{c}$, especially when it is necessary to distinguish it from that of lambda calculus $\left(\rightarrow_{\lambda}\right)$. We denote by $\stackrel{*}{\longrightarrow}$ the reflexive and transitive closure of $\rightarrow$. Note that arity is invariant under the relation $\rightarrow($ and $\stackrel{*}{\longrightarrow})$.

Example. Computation in $C C L M_{\beta}$.

Let $p l u s(x, y, z)=x+y+z$ be a function with 3 arguments giving their sum. In $C C L M_{\beta}$ this is translated to the following (the translation algorithm will be given in Section 5 ):

$$
\Lambda_{1}\left(\Lambda_{2}\left(\Lambda_{3}\left(p l u s \circ\left\langle p_{1}^{3}, p_{2}^{3}, p_{3}^{3}\right\rangle\right)\right)\right) \text {. }
$$

Now, we give only one value 2 to its first argument, and partially compute it using App. In the below, $2^{n}$ means the constant-valued function with $n$ arguments giving 2 as its result.

$$
\begin{aligned}
& \text { App } \circ\left\langle\Lambda_{1}\left(\Lambda_{2}\left(\Lambda_{3}\left(\text { plus } \circ\left\langle p_{1}^{3}, p_{2}^{3}, p_{3}^{3}\right\rangle\right)\right)\right), 2^{0}\right\rangle \\
& \rightarrow \Lambda_{2}\left(\Lambda_{3}\left(\text { plus } \circ\left\langle p_{1}^{3}, p_{2}^{3}, p_{3}^{3}\right\rangle\right)\right) \circ\left\langle 2^{0}\right\rangle \\
& \rightarrow \Lambda_{1}\left(\Lambda_{3}\left(\text { plus } \circ\left\langle p_{1}^{3}, p_{2}^{3}, p_{3}^{3}\right\rangle\right) \circ\left\langle 2^{0} \circ\langle\rangle^{1}, p_{1}^{1}\right\rangle\right) \\
& \rightarrow \Lambda_{1}\left(\Lambda_{3}\left(\text { plus } \circ\left\langle p_{1}^{3}, p_{2}^{3}, p_{3}^{3}\right\rangle\right) \circ\left\langle 2^{1}, p_{1}^{1}\right\rangle\right)
\end{aligned}
$$




$$
\begin{aligned}
& \left.\rightarrow \Lambda_{1}\left(\Lambda_{2}\left(\left(\text { plus } \circ\left\langle p_{1}^{3}, p_{2}^{3}, p_{3}^{3}\right\rangle\right) \circ\left\langle 2^{1} \circ\left\langle p_{1}^{2}\right\rangle, p_{1}^{1} \circ\left\langle p_{1}^{2}\right\rangle, p_{2}^{2}\right\rangle\right)\right) \quad \text { (by rules } 4,8\right) \\
& \stackrel{*}{\rightarrow} \Lambda_{1}\left(\Lambda_{2}\left(\left(\text { plus } \circ\left\langle p_{1}^{3}, p_{2}^{3}, p_{3}^{3}\right\rangle\right) \circ\left\langle 2^{2}, p_{1}^{2}, p_{2}^{2}\right\rangle\right)\right) \\
& \stackrel{*}{\rightarrow} \Lambda_{1}\left(\Lambda_{2}\left(\text { plus } \circ\left\langle 2^{2}, p_{1}^{2}, p_{2}^{2}\right\rangle\right)\right)
\end{aligned}
$$

\section{On models of $C C L M_{\beta}$.}

Before we examine the properties of $C C L M_{\beta}$ as a rewriting system, we digress and make a brief discussion about models of $C C L M_{\beta}$ as an equational system. Those who are interested only in the operational aspect of the system may skip this section.

Let $\mathbf{C}$ be a Cartesian closed category (ccc). We say that an object $u$ of $\mathbf{C}$ is reflexive, when there exists a pair of arrows $\phi: u \rightarrow u^{u}$ and $\psi: u^{u} \rightarrow u$ such that $\phi \circ \psi=i d_{u^{u}}$. It is known that ccc's with reflexive object are essentially the same as models of lambda calculus. See [2], [5], [8], [10], [11]. Similarly, ccc's with reflexive object characterize models of $C C L M_{\beta}$. We can naturally interpret terms of $C C L M_{\beta}$ in a ccc $\mathbf{C}$ with reflexive object $u$. Terms of arity $n$ in $C C L M_{\beta}$ are interpreted in the set $\mathbf{C}\left(u^{n}, u\right)$ of arrows from $u^{n}$ to $u$. Here $u^{n}$ denotes the product

$$
t \times u \times \cdots \times u \quad(n \text { times of } u),
$$

where $t$ is the terminal object of $\mathbf{C}$.

More formally, the interpretation of terms in $\mathbf{C}$ is the following. For each term $F$ of arity $\dot{n}$, we define the arrow $\llbracket F \rrbracket$ from $u^{n}$ to $u$ in $\mathbf{C}$ as follows. Here we assume that for every constant $f$ other than $p_{i}^{n}$ and $A p p, \llbracket f \rrbracket$ is already specified.

1. $\llbracket p_{i}^{n} \rrbracket=\pi_{i+1}^{t, u, \ldots, u} \quad\left(\right.$ the $(i+1)$-th projection from $u^{n}$ to $\left.u\right)$.

2. $\llbracket A p p \rrbracket=e v^{u, u} \circ\left(\phi \times i d_{u}\right)$, where $e v^{u, u}$ is the evaluation map from $u^{u}$ to $u$.

3. $\llbracket F \circ\left\langle G_{1}, \ldots, G_{n}\right\rangle \rrbracket=\llbracket F \rrbracket \circ\left\langle\llbracket G_{1} \rrbracket, \ldots, \llbracket G_{n} \rrbracket\right\rangle$.

4. $\llbracket \Lambda(F) \rrbracket=\psi \circ \Lambda_{u^{n}, u}\left(\llbracket F \rrbracket \circ \pi_{1}^{u^{n}, u}\right)$, 
where $\Lambda_{u^{n}, u}(h): u^{n} \rightarrow u^{u}$ is the transpose map of $h: u^{n} \times u \rightarrow u$.

Based on this interpretation, we can prove that, if $F=G$ in $C C L M_{\beta}$ as an equational system, then $\llbracket F \rrbracket=\llbracket G \rrbracket$ in $\mathbf{C}$. That is, $\mathbf{C}$ is a model of $C C L M_{\beta}$.

\section{Auxiliary combinators.}

We further introduce the derived combinators of currying and application in a more general form. First the intuitive meanings. For $m \geq 1$, the operator $\Lambda^{m}(-)$ means currying $m$ times. Thus, for an $n$-argument function $f, \Lambda_{n}^{m}(f)$ is an $(n-m)$-argument function which gives an $m$-argument function as a result. More precisely,

$$
\Lambda_{n}^{m}(f) \equiv \Lambda_{n-m+1}\left(\Lambda_{n-m+2}\left(\cdots\left(\Lambda_{n}(f)\right) \cdots\right)\right) .
$$

Informally, in a lambda-calculus like notation, $\Lambda_{n}^{m}(f)$ means

$$
\lambda\left\langle x_{1}, \ldots, x_{n-m}\right\rangle \cdot\left(\lambda x_{n-m+1} \ldots \lambda x_{n} . f\left\langle x_{1}, \ldots, x_{n}\right\rangle\right) .
$$

(We do not yet, and will not in this paper, formally define the angular brackets \langle\rangle in lambda calculus. The above expression is only for the reader's intuitive understanding.)

Likewise, for $m \geq 1, A p p^{m}$ receives $m+1$ arguments, an $n$-argument function, $(n \geq m)$, and $m$ values; it implies applying the values as the first $m$ arguments of the function, and returns a function of $n-m$ arguments. Thus, in particular, for an $n$-argument function, $A p p^{n}$ is the usual (full) application. $A p p^{m}$ is informally represented by

$$
\lambda\left\langle z, x_{1}, \ldots, x_{m}\right\rangle . z x_{1} \ldots x_{m} .
$$

Now we formally introduce auxiliary combinators.

(1) For $n \geq 0$, define $i d^{n} \equiv\left\langle p_{1}^{n}, \ldots, p_{n}^{n}\right\rangle^{n}$.

(2) For $m \geq 0$ and $n \geq 0$, define $\pi_{1}^{m, n} \equiv\left\langle p_{1}^{m+n}, \ldots, p_{m}^{m+n}\right\rangle^{m+n}$, and $\pi_{2}^{m, n} \equiv\left\langle p_{m+1}^{m+n}, \ldots, p_{m+n}^{m+n}\right\rangle^{m+n}$.

(3) For $n \geq 1,1 \leq m \leq n$, and $F$ a term of arity $n$, define the $(n-m)$-ary term $\Lambda^{m}(F)$ inductively by $\Lambda^{1}(F) \equiv \Lambda(F)$, and $\Lambda^{m+1}(F) \equiv \Lambda\left(\Lambda^{m}(F)\right)$.

(4) For $m \geq 1$ define 


$$
A p p^{m} \equiv A p p \circ\left\langle A p p \circ\left\langle\cdots\left\langle A p p \circ\left\langle A p p \circ\left\langle p_{1}^{m+1}, p_{2}^{m+1}\right\rangle, p_{3}^{m+1}\right\rangle, p_{4}^{m+1}\right\rangle, \cdots\right\rangle, p_{m+1}^{m+1}\right\rangle,
$$

( $m$ times of $A p p)$.

Remark 1. For $m \geq 1$ and $n \geq 1$,

$$
\Lambda^{m}\left(\Lambda^{n}(F)\right) \equiv \Lambda^{m+n}(F)
$$

\section{Remark 2.}

For $m \geq 1$ and $n$-ary terms $F, G_{1}, \ldots, G_{m}$, define the $n$-ary term $A P P^{m}\left\{F, G_{1}, \ldots, G_{m}\right\}$ inductively by

$$
A P P^{1}\left\{F, G_{1}\right\} \equiv A p p \circ\left\langle F, G_{1}\right\rangle
$$

and

$$
A P P^{m+1}\left\{F, G_{1}, \ldots, G_{m+1}\right\} \equiv A p p \circ\left\langle A P P^{m}\left\{F, G_{1}, \ldots, G_{m}\right\}, G_{m+1}\right\rangle .
$$

Then we easily have:

$$
A p p^{m} \equiv A P P^{m}\left\{p_{1}^{m+1}, p_{2}^{m+1}, \ldots, p_{m+1}^{m+1}\right\}
$$

$$
A p p^{m} \circ\left\langle F, G_{1}, \ldots, G_{m}\right\rangle \stackrel{*}{\longrightarrow} A P P^{m}\left\{F, G_{1}, \ldots, G_{m}\right\}
$$

$$
A P P^{m}\left\{A P P^{l}\left\{F, G_{1}, \ldots, G_{l}\right\}, H_{1}, \ldots, H_{m}\right\} \equiv A P P^{l+m}\left\{F, G_{1}, \ldots, G_{l}, H_{1}, \ldots, H_{m}\right\} \text {. }
$$

Rules 4 and 5 of $C C L M_{\beta}$ have natural extensions for $\Lambda^{m}$ and $A p p^{m}$, which are the following propositions.

Proposition 4.1. Let $F$ be $(m+n)$-ary and $G_{1}, \ldots, G_{n}$ be $k$-ary, where $m \geq 1$. Then

$$
\begin{aligned}
\Lambda_{m+n}^{m}(F) & \circ\left\langle G_{1}, \ldots, G_{n}\right\rangle^{k} \\
& \stackrel{*}{\longrightarrow} \Lambda_{k+m}^{m}\left(F \circ\left\langle G_{1} \circ \pi_{1}^{k, m}, \ldots, G_{n} \circ \pi_{1}^{k, m}, p_{k+1}^{k+m}, \ldots, p_{k+m}^{k+m}\right\rangle^{k+m}\right) .
\end{aligned}
$$

Proof. Induction on $m$. When $m=1$ this is identical to rule 4 of $C C L M_{\beta}$.

$$
\begin{aligned}
& \Lambda^{m+1}(F) \circ\left\langle G_{1}, \ldots, G_{n}\right\rangle \\
& \rightarrow \Lambda\left(\Lambda^{m}(F) \circ\left\langle G_{1} \circ \pi_{1}^{k, 1}, \ldots, G_{n} \circ \pi_{1}^{k, 1}, p_{k+1}^{k+1}\right\rangle\right) \\
& \stackrel{*}{\rightarrow} \Lambda\left(\Lambda ^ { m } \left(F \circ \left\langle\left(G_{1} \circ \pi_{1}^{k, 1}\right) \circ \pi_{1}^{k+1, m}, \ldots,\left(G_{n} \circ \pi_{1}^{k, 1}\right) \circ \pi_{1}^{k+1, m}, p_{k+1}^{k+1} \circ \pi_{1}^{k+1, m},\right.\right.\right. \\
& \left.\left.\left.\quad p_{k+2}^{k+1+m}, \ldots, p_{k+1+m}^{k+1+m}\right\rangle\right)\right) \\
& \stackrel{*}{\rightarrow} \Lambda^{m+1}\left(F \circ\left\langle G_{1} \circ \pi_{1}^{k, m+1}, \ldots, G_{n} \circ \pi_{1}^{k, m+1}, p_{k+1}^{k+m+1}, \ldots, p_{k+m+1}^{k+m+1}\right\rangle\right) .
\end{aligned}
$$

Proposition 4.2. Let $F$ be $(m+n)$-ary, and $G_{1}, \ldots, G_{m}$ be $n$-ary, where $m \geq 1$. Then,

$$
A P P^{m}\left\{\Lambda_{m+n}^{m}(F), G_{1}, \ldots, G_{m}\right\} \stackrel{*}{\longrightarrow} F \circ\left\langle p_{1}^{n}, \ldots, p_{n}^{n}, G_{1}, \ldots, G_{m}\right\rangle^{n} .
$$

Proof. By induction on $m$. When $m=1$ this is rule 5 .

$$
\begin{aligned}
& A P P^{m+1}\left\{\Lambda^{m+1}(F), G_{1}, \ldots, G_{m}, G_{m+1}\right\} \\
& \equiv A p p \circ\left\langle A P P^{m}\left\{\Lambda^{m+1}(F), G_{1}, \ldots, G_{m}\right\}, G_{m+1}\right\rangle \\
& \stackrel{*}{\rightarrow} A p p \circ\left\langle\Lambda(F) \circ\left\langle p_{1}^{n}, \ldots, p_{n}^{n}, G_{1}, \ldots, G_{m}\right\rangle, G_{m+1}\right\rangle \quad \text { (by induction hypothesis) }
\end{aligned}
$$




$$
\begin{aligned}
& \rightarrow A p p \circ\left\langle\Lambda\left(F \circ\left\langle p_{1}^{n} \circ \pi_{1}^{n, 1}, \ldots, p_{n}^{n} \circ \pi_{1}^{n, 1}, G_{1} \circ \pi_{1}^{n, 1}, \ldots, G_{m} \circ \pi_{1}^{n, 1}, p_{n+1}^{n+1}\right\rangle\right), G_{m+1}\right\rangle \\
& \stackrel{*}{*} A p p \circ\left\langle\Lambda\left(F \circ\left\langle p_{1}^{n+1}, \ldots, p_{n}^{n+1}, G_{1} \circ \pi_{1}^{n, 1}, \ldots, G_{m} \circ \pi_{1}^{n, 1}, p_{n+1}^{n+1}\right\rangle\right), G_{m+1}\right\rangle \\
& \rightarrow\left(F \circ\left\langle p_{1}^{n+1}, \ldots, p_{n}^{n+1}, G_{1} \circ \pi_{1}^{n, 1}, \ldots, G_{m} \circ \pi_{1}^{n, 1}, p_{n+1}^{n+1}\right\rangle\right) \circ\left\langle p_{1}^{n}, \ldots, p_{n}^{n}, G_{m+1}\right\rangle \\
& \stackrel{*}{\rightarrow} F \circ\left\langle p_{1}^{n}, \ldots, p_{n}^{n}, G_{1}, \ldots, G_{m}, G_{m+1}\right\rangle .
\end{aligned}
$$

Proposition 4.3. Let $F$ be $(m+n)$-ary, and $G_{1}, \ldots, G_{m}$ be $n$-ary, where $m \geq 1$. Then,

$$
\operatorname{App}^{m} \circ\left\langle\Lambda_{m+n}^{m}(F), G_{1}, \ldots, G_{m}\right\rangle^{n} \stackrel{*}{\longrightarrow} F \circ\left\langle p_{1}^{n}, \ldots, p_{n}^{n}, G_{1}, \ldots, G_{m}\right\rangle^{n} .
$$

Proof. Immediate by combining ii) of remark 2 above and Proposition 4.2.

The auxiliary combinators will be useful in actual computations in $C C L M_{\beta}$, since, as the example below indicates, they can be used to shorten the length of computation.

Example. Computation in $C C L M_{\beta}$ with the auxiliary combinators.

Let us use the same function $p l u s(x, y, z)=x+y+z$, and give two values 2 and 3 to it:

$$
\begin{aligned}
& A p p^{2} \circ\left\langle\Lambda^{3}\left(p l u s \circ\left\langle p_{1}^{3}, p_{2}^{3}, p_{3}^{3}\right\rangle\right), 2^{0}, 3^{0}\right\rangle \\
& \stackrel{*}{\rightarrow} \Lambda\left(\text { plus } \circ\left\langle p_{1}^{3}, p_{2}^{3}, p_{3}^{3}\right\rangle\right) \circ\left\langle 2^{0}, 3^{0}\right\rangle \\
& \rightarrow \Lambda\left(\left(\text { plus } \circ\left\langle p_{1}^{3}, p_{2}^{3}, p_{3}^{3}\right\rangle\right) \circ\left\langle 2^{0} \circ\langle\rangle^{1}, 3^{0} \circ\langle\rangle^{1}, p_{1}^{1}\right\rangle\right) \\
& \stackrel{*}{\rightarrow} \Lambda\left(\text { plus } \circ\left\langle 2^{1}, 3^{1}, p_{1}^{1}\right\rangle\right) .
\end{aligned}
$$




\section{Translations between $C C L M_{\beta}$ and lambda calculus.}

In this section we define translation algorithms for both directions between $C C L M_{\beta}$ and lambda calculus, and we establish the natural relationship between the terms in these two systems. The lambda calculus we are concerned is, more specifically, the type-free $\lambda_{\beta}$-calculus (without product), which we will denote by $\lambda$. We assume that $\lambda$ contains constants. Moreover, we assume that there is given a one-to-one correspondence between the constants in $C C L M_{\beta}$ other than $p_{i}^{n}$ and $A p p$, and the constants in $\lambda$.

Firstly, the translation algorithm from $\lambda$ to $C C L M_{\beta}$ is described. For terms $M$ and $N$ in $\lambda$, we denote by $M[x:=N]$ the $\lambda$-term obtained by substituting $N$ for each occurrence of a free variable $x$ in $M$.

Convention. Let $\gamma \equiv\left\langle z_{1}, \ldots, z_{n}\right\rangle$ be a sequence of distinct variables $z_{1}, \ldots, z_{n}, n \geq 0$. For such a sequence $\gamma$ and a variable $x$, we denote by $\gamma x$ the sequence of the elements of $\gamma$ followed by $x$, that is, $\gamma x \equiv\left\langle z_{1}, \ldots, z_{n}, x\right\rangle$. Similarly, for two sequences $\alpha \equiv\left\langle x_{1}, \ldots, x_{l}\right\rangle$ and $\beta \equiv\left\langle y_{1}, \ldots, y_{m}\right\rangle$, we denote $\alpha \beta \equiv\left\langle x_{1}, \ldots, x_{l}, y_{1}, \ldots, y_{m}\right\rangle$.

Definition. For each term $M$ in $\lambda$ whose free variables are contained in $\gamma \equiv\left\langle z_{1}, \ldots, z_{n}\right\rangle$, we define inductively a term of arity $n$ in $C C L M_{\beta}$, denoted by $[\lambda \gamma \cdot M]$, as follows:

1. $\left[\lambda \gamma . z_{i}\right] \equiv p_{i}^{n}, 1 \leq i \leq n$.

2. $[\lambda \gamma . c] \equiv \Lambda^{s}\left(c \circ\left\langle p_{n+1}^{n+s}, \ldots, p_{n+s}^{n+s}\right\rangle\right)$, where $c$ is a constant of arity $s, s \geq 1$. When $s=0$, we define $[\lambda \gamma . c] \equiv c \circ\langle\rangle^{n}$.

3. $[\lambda \gamma \cdot(\lambda x . M)] \equiv \Lambda\left(\left[\lambda \gamma x^{\prime} . M\left[x:=x^{\prime}\right]\right]\right)$, where $x^{\prime} \equiv x$ if $x$ is not in $\gamma$, otherwise $x^{\prime}$ is a new variable.

4. $\left[\lambda \gamma . c M_{1} \ldots M_{s}\right] \equiv c \circ\left\langle\left[\lambda \gamma \cdot M_{1}\right], \ldots,\left[\lambda \gamma \cdot M_{s}\right]\right\rangle^{n}$, where $c$ is a constant of arity $s, s \geq 0$.

5. $\left[\lambda \gamma \cdot M_{1} M_{2}\right] \equiv A p p \circ\left\langle\left[\lambda \gamma \cdot M_{1}\right],\left[\lambda \gamma \cdot M_{2}\right]\right\rangle$, where $M_{1} M_{2}$ is not of the form in 4 .

In the following discussions, whenever we mention $\left[\lambda\left\langle z_{1}, \ldots, z_{n}\right\rangle \cdot M\right]$, we assume that the variables $z_{1}, \ldots, z_{n}$ are distinct and that all the free variables in $M$ are contained in the set $\left\{z_{1}, \ldots, z_{n}\right\}$.

Remark. The above definition of translation for constants of case 4 may seem somewhat artificial. Indeed, a different definition of translation without case 4 (that is, case 2 only) would be simpler, and actually almost sufficient. Under this simpler definition, 
the righthand-side of the translation of case 4 is obtained as a result of reductions for the translated term of the lefthand-side (inspect the proof of Proposition 5.1 below). However, in Theorem 6.3 of the next section (precisely, in case 3 of its proof), we need our present translation for constants.

We give the general case of the translation of constants from $\lambda$ to $C C L M_{\beta}$.

Proposition 5.1. Let $c$ be an $s$-ary constant in $\lambda$, and $0<m<s$. Let $\gamma \equiv\left\langle z_{1}, \ldots, z_{n}\right\rangle$. Then we have:

$$
\begin{aligned}
& {\left[\lambda \gamma \cdot c M_{1} \ldots M_{m}\right]} \\
& \stackrel{*}{\longrightarrow} c \Lambda^{s-m}\left(c \circ\left\langle\left[\lambda \gamma \cdot M_{1}\right] \circ \pi_{1}^{n, s-m}, \ldots,\left[\lambda \gamma \cdot M_{m}\right] \circ \pi_{1}^{n, s-m}, p_{n+1}^{n+s-m}, \ldots, p_{n+s-m}^{n+s-m}\right\rangle\right) .
\end{aligned}
$$

Proof. $\quad\left[\lambda \gamma . c M_{1} \ldots M_{m}\right]$

$$
\begin{aligned}
& \equiv A p p \circ\left\langle A p p \circ\left\langle\cdots\left\langle A p p \circ\left\langle[\lambda \gamma \cdot c],\left[\lambda \gamma \cdot M_{1}\right]\right\rangle,\left[\lambda \gamma \cdot M_{2}\right]\right\rangle \cdots\right\rangle,\left[\lambda \gamma \cdot M_{m}\right]\right\rangle \\
& \equiv A P P^{m}\left\{[\lambda \gamma \cdot c],\left[\lambda \gamma \cdot M_{1}\right], \ldots,\left[\lambda \gamma \cdot M_{m}\right]\right\} \\
& \equiv A P P^{m}\left\{\Lambda^{s}\left(c \circ \pi_{2}^{n, s}\right),\left[\lambda \gamma \cdot M_{1}\right], \ldots,\left[\lambda \gamma \cdot M_{m}\right]\right\} \\
& \stackrel{*}{\rightarrow} \Lambda^{s-m}\left(c \circ \pi_{2}^{n, s}\right) \circ\left\langle p_{1}^{n}, \ldots, p_{n}^{n},\left[\lambda \gamma \cdot M_{1}\right], \ldots,\left[\lambda \gamma \cdot M_{m}\right]\right\rangle \quad \quad \text { (by Proposition 4.2) } \\
& \stackrel{*}{\rightarrow} \Lambda^{s-m}\left(( c \circ \pi _ { 2 } ^ { n , s } ) \circ \left\langlep_{1}^{n} \circ \pi_{1}^{n, s-m}, \ldots, p_{n}^{n} \circ \pi_{1}^{n, s-m},\right.\right. \\
& \left.\left.\quad\left[\lambda \gamma \cdot M_{1}\right] \circ \pi_{1}^{n, s-m}, \ldots,\left[\lambda \gamma \cdot M_{m}\right] \circ \pi_{1}^{n, s-m}, p_{n+1}^{n+s-m}, \ldots, p_{n+s-m}^{n+s-m}\right\rangle\right) \\
& \quad \text { (by Proposition 4.1) } \\
& \stackrel{*}{\rightarrow} \Lambda^{s-m}\left(c \circ\left\langle\left[\lambda \gamma \cdot M_{1}\right] \circ \pi_{1}^{n, s-m}, \ldots,\left[\lambda \gamma \cdot M_{m}\right] \circ \pi_{1}^{n, s-m}, p_{n+1}^{n+s-m}, \ldots, p_{n+s-m}^{n+s-m}\right\rangle\right) .
\end{aligned}
$$

Remark. If we use Lemma 5.3 which will be established soon, the righthand-side of the rewriting of the above proposition can be transformed further:

(the last term of the proof)

$$
\begin{gathered}
\stackrel{*}{\rightarrow} \Lambda^{s-m}\left(c \circ \left\langle\left[\lambda \gamma x_{1} \ldots x_{s-m} \cdot M_{1}\right], \ldots,\left[\lambda \gamma x_{1} \ldots x_{s-m} \cdot M_{m}\right],\right.\right. \\
\left.\left.\quad\left[\lambda \gamma x_{1} \ldots x_{s-m} \cdot x_{1}\right], \ldots,\left[\lambda \gamma x_{1} \ldots x_{s-m} \cdot x_{s-m}\right]\right\rangle\right) \\
\equiv \Lambda^{s-m}\left(\left[\lambda \gamma x_{1} \ldots x_{s-m} . c M_{1} \ldots M_{m} x_{1} \ldots x_{s-m}\right]\right)
\end{gathered}
$$

Next, we give the translation algorithm from $C C L M_{\beta}$ to $\lambda$.

Definition. For each term $F$ of arity $n$ in $C C L M_{\beta}$ and terms $N_{1}, \ldots, N_{n}$ of $\lambda$ we define the term $F^{*}\left[N_{1}, \ldots, N_{n}\right]$ in $\lambda$, inductively by the structure of $F$, as follows.

1. $\left(p_{i}^{n}\right)^{*}\left[N_{1}, \ldots, N_{n}\right] \equiv N_{i}$.

2. $A p p^{*}\left[N_{1}, N_{2}\right] \equiv N_{1} N_{2}$.

3. $f^{*}\left[N_{1}, \ldots, N_{n}\right] \equiv f N_{1} \ldots N_{n}$,

for each $n$-ary constant $f$ other than $p_{i}^{n}$ and $A p p$. 
4. $\left(F \circ\left\langle G_{1}, \ldots, G_{m}\right\rangle^{n}\right)^{*}\left[N_{1}, \ldots, N_{n}\right] \equiv F^{*}\left[G_{1}^{*}\left[N_{1}, \ldots, N_{n}\right], \ldots, G_{m}^{*}\left[N_{1}, \ldots, N_{n}\right]\right]$.

5. $(\Lambda(F))^{*}\left[N_{1}, \ldots, N_{n}\right] \equiv \lambda x .\left(F^{*}\left[N_{1}, \ldots, N_{n}, x\right]\right)$,

where $x$ is a variable not free in $N_{1}, \ldots, N_{n}$.

A term $F$ of arity $n$ in $C C L M_{\beta}$ means an $n$-ary function. Thus $F$ is intuitively represented by a $\lambda$-term $M$ with free variables $x_{1}, \ldots, x_{n}$. In the above definition $F^{*}\left[N_{1}, \ldots, N_{n}\right]$ means $M\left[x_{1}:=N_{1}, \ldots, x_{n}:=N_{n}\right]$.

Now we return to the former translation algorithm and give three basic lemmas concerning it.

Lemma 5.2. $\left[\lambda\left\langle z_{1}, \ldots, z_{n}\right\rangle \cdot M\right] \equiv\left[\lambda\left\langle z_{1}^{\prime}, \ldots, z_{n}^{\prime}\right\rangle \cdot M\left[z_{1}:=z_{1}^{\prime}, \ldots, z_{n}:=z_{n}^{\prime}\right]\right]$.

Proof. Easy and omitted.

Lemma 5.3. Let $\alpha=\left\langle x_{1}, \ldots, x_{l}\right\rangle, \beta=\left\langle y_{1}, \ldots, y_{m}\right\rangle$, and $\gamma=\left\langle z_{1}, \ldots, z_{n}\right\rangle$. Then,

$$
[\lambda \alpha \gamma \cdot M] \circ\left\langle p_{1}^{l+m+n}, \ldots, p_{l}^{l+m+n}, p_{l+m+1}^{l+m+n}, \ldots, p_{l+m+n}^{l+m+n}\right\rangle \stackrel{*}{\longrightarrow}_{c}[\lambda \alpha \beta \gamma . M]
$$

As a special case, we have

$$
[\lambda \alpha . M] \circ\left\langle p_{1}^{l+m}, \ldots, p_{l}^{l+m}\right\rangle \stackrel{*}{\longrightarrow}_{c}[\lambda \alpha \beta . M] .
$$

Proof. The proof is by induction on the structure of $M$.

Case $1 . M \equiv x_{i}, 1 \leq i \leq l$.

$$
\begin{aligned}
& {\left[\lambda \alpha \gamma \cdot x_{i}\right] \circ\left\langle p_{1}^{l+m+n}, \ldots, p_{l}^{l+m+n}, p_{l+m+1}^{l+m+n}, \ldots, p_{l+m+n}^{l+m+n}\right\rangle} \\
& \equiv p_{i}^{l+n} \circ\left\langle p_{1}^{l+m+n}, \ldots, p_{l}^{l+m+n}, p_{l+m+1}^{l+m+n}, \ldots, p_{l+m+n}^{l+m+n}\right\rangle \\
& \rightarrow p_{i}^{l+m+n} \\
& \equiv\left[\lambda \alpha \beta \gamma \cdot x_{i}\right] .
\end{aligned}
$$

Case 2. $M \equiv z_{k}, 1 \leq k \leq n$.

Similar to case 1 .

Case 3. $M \equiv c$ (s-ary constant).

When $s=0$ it is clear. Suppose $s \geq 1$.

$$
\begin{aligned}
& {[\lambda \alpha \gamma . c] \circ\left\langle p_{1}^{l+m+n}, \ldots, p_{l}^{l+m+n}, p_{l+m+1}^{l+m+n}, \ldots, p_{l+m+n}^{l+m+n}\right\rangle} \\
& \equiv \Lambda^{s}\left(c \circ \pi_{2}^{l+n, s}\right) \circ\left\langle p_{1}^{l+m+n}, \ldots, p_{l}^{l+m+n}, p_{l+m+1}^{l+m+n}, \ldots, p_{l+m+n}^{l+m+n}\right\rangle \\
& \stackrel{*}{\rightarrow} \Lambda^{s}\left(( c \circ \pi _ { 2 } ^ { l + n , s } ) \circ \left\langlep_{1}^{l+m+n} \circ \pi_{1}^{l+m+n, s}, \ldots, p_{l}^{l+m+n} \circ \pi_{1}^{l+m+n, s},\right.\right. \\
& \left.\left.\quad p_{l+m+1}^{l+m+n} \circ \pi_{1}^{l+m+n, s}, \ldots, p_{l+m+n}^{l+m+n} \circ \pi_{1}^{l+m+n, s}, p_{l+m+n+1}^{l+m+n+s}, \ldots, p_{l+m+n+s}^{l+m+n+s}\right\rangle\right)
\end{aligned}
$$

$\stackrel{*}{\rightarrow} \Lambda^{s}\left(c \circ \pi_{2}^{l+m+n, s}\right)$

(by Proposition 4.1)

$\equiv[\lambda \alpha \beta \gamma . c]$. 
Case 4. $M \equiv \lambda w \cdot M_{1}$.

$$
\begin{aligned}
& {\left[\lambda \alpha \gamma \cdot\left(\lambda w \cdot M_{1}\right)\right] \circ\left\langle p_{1}^{l+m+n}, \ldots, p_{l}^{l+m+n}, p_{l+m+1}^{l+m+n}, \ldots, p_{l+m+n}^{l+m+n}\right\rangle} \\
& \equiv \Lambda\left(\left[\lambda \alpha \gamma w^{\prime} \cdot M_{1}\left[w:=w^{\prime}\right]\right]\right) \circ\left\langle p_{1}^{l+m+n}, \ldots, p_{l}^{l+m+n}, p_{l+m+n}^{l+m}, \ldots, p_{l+m+n}^{l+m+n}\right\rangle \\
& \rightarrow \Lambda\left([ \lambda \alpha \gamma w ^ { \prime } \cdot M _ { 1 } [ w : = w ^ { \prime } ] ] \circ \left\langlep_{1}^{l+m+n} \circ \pi_{1}^{l+m+n, 1}, \ldots, p_{l}^{l+m+n} \circ \pi_{1}^{l+m+n, 1},\right.\right. \\
& \left.\left.\quad p_{l+m+1}^{l+m+n} \circ \pi_{1}^{l+m+n, 1}, \ldots, p_{l+m+n}^{l+m+n} \circ \pi_{1}^{l+m+n, 1}, p_{l+m+n+1}^{l+m+n+1}\right\rangle\right) \\
& \stackrel{*}{\rightarrow} \Lambda\left([ \lambda \alpha \gamma w ^ { \prime } \cdot M _ { 1 } [ w : = w ^ { \prime } ] ] \circ \left\langlep_{1}^{l+m+n+1}, \ldots, p_{l}^{l+m+n+1},\right.\right. \\
& \left.\left.\quad p_{l+m+1}^{l+m+n+1}, \ldots, p_{l+m+n}^{l+m+n+1}, p_{l+m+n+1}^{l+m+n+1}\right\rangle\right) \\
& \stackrel{*}{\rightarrow} \Lambda\left(\left[\lambda \alpha \beta \gamma w^{\prime} \cdot M_{1}\left[w:=w^{\prime}\right]\right]\right) \quad \text { (by induction hypothesis) } \\
& \equiv\left[\lambda \alpha \beta \gamma \cdot\left(\lambda w \cdot M_{1}\right)\right] .
\end{aligned}
$$

Case 5. $M \equiv c M_{1} \ldots M_{s}$ ( $c$ is an $s$-ary constant).

$\left[\lambda \alpha \gamma . c M_{1} \ldots M_{s}\right] \circ\left\langle p_{1}^{l+m+n}, \ldots, p_{l}^{l+m+n}, p_{l+m+1}^{l+m+n}, \ldots, p_{l+m+n}^{l+m+n}\right\rangle$

$\equiv\left(c \circ\left\langle\left[\lambda \alpha \gamma \cdot M_{1}\right], \ldots,\left[\lambda \alpha \gamma \cdot M_{s}\right]\right\rangle\right) \circ\left\langle p_{1}^{l+m+n}, \ldots, p_{l}^{l+m+n}, p_{l+m+1}^{l+m+n}, \ldots, p_{l+m+n}^{l+m+n}\right\rangle$

$\rightarrow c \circ\left\langle\left[\lambda \alpha \gamma \cdot M_{1}\right] \circ\left\langle p_{1}^{l+m+n}, \ldots, p_{l}^{l+m+n}, p_{l+m+1}^{l+m+n}, \ldots, p_{l+m+n}^{l+m+n}\right\rangle, \ldots\right.$,

$$
\left.\left[\lambda \alpha \gamma \cdot M_{s}\right] \circ\left\langle p_{1}^{l+m+n}, \ldots, p_{l}^{l+m+n}, p_{l+m+1}^{l+m+n}, \ldots, p_{l+m+n}^{l+m+n}\right\rangle\right\rangle
$$

(by rule 1)

$\stackrel{*}{\rightarrow} c \circ\left\langle\left[\lambda \alpha \beta \gamma \cdot M_{1}\right], \ldots,\left[\lambda \alpha \beta \gamma \cdot M_{s}\right]\right\rangle \quad$ (by induction hypothesis)

$\equiv\left[\lambda \alpha \beta \gamma . c M_{1} \ldots M_{s}\right]$.

Case 6. $M \equiv M_{1} M_{2}$, and $M$ is not of the form of case 5 .

$\left[\lambda \alpha \gamma \cdot M_{1} M_{2}\right] \circ\left\langle p_{1}^{l+m+n}, \ldots, p_{l}^{l+m+n}, p_{l+m+1}^{l+m+n}, \ldots, p_{l+m+n}^{l+m+n}\right\rangle$

$\equiv\left(A p p \circ\left\langle\left[\lambda \alpha \gamma \cdot M_{1}\right],\left[\lambda \alpha \gamma \cdot M_{2}\right]\right\rangle\right) \circ\left\langle p_{1}^{l+m+n}, \ldots, p_{l}^{l+m+n}, p_{l+m+1}^{l+m+n}, \ldots, p_{l+m+n}^{l+m+n}\right\rangle$

$\stackrel{*}{\rightarrow} A p p \circ\left\langle\left[\lambda \alpha \beta \gamma \cdot M_{1}\right],\left[\lambda \alpha \beta \gamma \cdot M_{2}\right]\right\rangle \quad$ (by induction hypothesis)

$\equiv\left[\lambda \alpha \beta \gamma \cdot M_{1} M_{2}\right]$.

Lemma 5.4. Let $\alpha=\left\langle x_{1}, \ldots, x_{m}\right\rangle$, and $\gamma=\left\langle z_{1}, \ldots, z_{n}\right\rangle$. Then

$$
[\lambda \alpha . M] \circ\left\langle\left[\lambda \gamma \cdot N_{1}\right], \ldots,\left[\lambda \gamma \cdot N_{m}\right]\right\rangle \stackrel{*}{\longrightarrow}_{c}\left[\lambda \gamma \cdot M\left[x_{1}:=N_{1}, \ldots, x_{m}:=N_{m}\right]\right] .
$$

Proof. By induction on the structure of $M$.

Case $1 . M \equiv x_{i}, 1 \leq i \leq m$.

$$
\begin{aligned}
& {\left[\lambda \alpha \cdot x_{i}\right] \circ\left\langle\left[\lambda \gamma \cdot N_{1}\right], \ldots,\left[\lambda \gamma \cdot N_{m}\right]\right\rangle} \\
& \equiv p_{i}^{m} \circ\left\langle\left[\lambda \gamma \cdot N_{1}\right], \ldots,\left[\lambda \gamma \cdot N_{m}\right]\right\rangle \\
& \rightarrow\left[\lambda \gamma \cdot N_{i}\right] .
\end{aligned}
$$

Case 2. $M \equiv c \quad(c$ is an $s$-ary constant $)$.

When $s=0$ it is clear. Suppose $s \geq 1$.

$[\lambda \alpha . c] \circ\left\langle\left[\lambda \gamma . N_{1}\right], \ldots,\left[\lambda \gamma . N_{m}\right]\right\rangle$

$\equiv \Lambda^{s}\left(c \circ \pi_{2}^{m, s}\right) \circ\left\langle\left[\lambda \gamma . N_{1}\right], \ldots,\left[\lambda \gamma . N_{m}\right]\right\rangle$

$\stackrel{*}{\rightarrow} \Lambda^{s}\left(\left(c \circ \pi_{2}^{m, s}\right) \circ\left\langle\left[\lambda \gamma . N_{1}\right] \circ \pi_{1}^{n, s}, \ldots,\left[\lambda \gamma . N_{m}\right] \circ \pi_{1}^{n, s}, p_{n+1}^{n+s}, \ldots, p_{n+s}^{n+s}\right\rangle\right)$ 
(by Proposition 4.1)

$$
\begin{aligned}
& \stackrel{*}{\rightarrow} \Lambda^{s}\left(c \circ \pi_{2}^{n, s}\right) \\
& \equiv[\lambda \gamma . c] .
\end{aligned}
$$

Case 3. $M \equiv \lambda x \cdot M_{1}$.

$$
\begin{aligned}
& {\left[\lambda \alpha \cdot\left(\lambda x \cdot M_{1}\right)\right] \circ\left\langle\left[\lambda \gamma \cdot N_{1}\right], \ldots,\left[\lambda \gamma \cdot N_{m}\right]\right\rangle} \\
& \equiv \Lambda\left(\left[\lambda \alpha x^{\prime} . M_{1}\left[x:=x^{\prime}\right]\right]\right) \circ\left\langle\left[\lambda \gamma \cdot N_{1}\right], \ldots,\left[\lambda \gamma \cdot N_{m}\right]\right\rangle \\
& \rightarrow \Lambda\left(\left[\lambda \alpha x^{\prime} . M_{1}\left[x:=x^{\prime}\right]\right] \circ\left\langle\left[\lambda \gamma \cdot N_{1}\right] \circ \pi_{1}^{n, 1}, \ldots,\left[\lambda \gamma \cdot N_{m}\right] \circ \pi_{1}^{n, 1}, p_{n+1}^{n+1}\right\rangle\right) \\
& \stackrel{*}{\rightarrow} \Lambda\left(\left[\lambda \alpha x^{\prime} . M_{1}\left[x:=x^{\prime}\right]\right] \circ\left\langle\left[\lambda \gamma x^{\prime} . N_{1}\right], \ldots,\left[\lambda \gamma x^{\prime} . N_{m}\right],\left[\lambda \gamma x^{\prime} . x^{\prime}\right]\right\rangle\right)
\end{aligned}
$$

(by Lemma 5.3)

$\stackrel{*}{\rightarrow} \Lambda\left(\left[\lambda \gamma x^{\prime} . M_{1}\left[x_{1}:=N_{1}, \ldots, x_{m}:=N_{m}, x:=x^{\prime}\right]\right]\right)$

(by induction hypothesis)

$$
\equiv\left[\lambda \gamma \cdot\left(\lambda x . M_{1}\right)\left[x_{1}:=N_{1}, \ldots, x_{m}:=N_{m}\right]\right] .
$$

Case 4. $M \equiv c M_{1} \ldots M_{s} \quad$ ( $c$ is an $s$-ary constant).

$$
\begin{aligned}
& {\left[\lambda \alpha \cdot c M_{1} \ldots M_{s}\right] \circ\left\langle\left[\lambda \gamma \cdot N_{1}\right], \ldots,\left[\lambda \gamma \cdot N_{m}\right]\right\rangle} \\
& \equiv\left(c \circ\left\langle\left[\lambda \alpha \cdot M_{1}\right], \ldots,\left[\lambda \alpha \cdot M_{s}\right]\right\rangle\right) \circ\left\langle\left[\lambda \gamma \cdot N_{1}\right], \ldots,\left[\lambda \gamma \cdot N_{m}\right]\right\rangle \\
& \rightarrow c \circ\left\langle\left[\lambda \alpha \cdot M_{1}\right] \circ\left\langle\left[\lambda \gamma \cdot N_{1}\right], \ldots,\left[\lambda \gamma \cdot N_{m}\right]\right\rangle, \ldots,\left[\lambda \alpha \cdot M_{s}\right] \circ\left\langle\left[\lambda \gamma \cdot N_{1}\right], \ldots,\left[\lambda \gamma \cdot N_{m}\right]\right\rangle\right\rangle \\
& \stackrel{*}{\rightarrow} c \circ\left\langle\left[\lambda \gamma \cdot M_{1}\left[x_{1}:=N_{1}, \ldots, x_{m}:=N_{m}\right]\right], \ldots,\left[\lambda \gamma \cdot M_{s}\left[x_{1}:=N_{1}, \ldots, x_{m}:=N_{m}\right]\right]\right\rangle \\
& \equiv\left[\lambda \gamma . c M_{1} \ldots M_{s}\left[x_{1}:=N_{1}, \ldots, x_{m}:=N_{m}\right]\right] .
\end{aligned}
$$

Case 5. $M \equiv M_{1} M_{2}$, and $M$ is not of the form of case 4 .

$$
\begin{aligned}
& {\left[\lambda \alpha \cdot M_{1} M_{2}\right] \circ\left\langle\left[\lambda \gamma \cdot N_{1}\right], \ldots,\left[\lambda \gamma \cdot N_{m}\right]\right\rangle} \\
& \equiv\left(A p p \circ\left\langle\left[\lambda \alpha \cdot M_{1}\right],\left[\lambda \alpha \cdot M_{2}\right]\right\rangle\right)\left\langle\left\langle\lambda \gamma \cdot N_{1}\right], \ldots,\left[\lambda \gamma \cdot N_{m}\right]\right\rangle \\
& \rightarrow A p p \circ\left\langle\left[\lambda \alpha \cdot M_{1}\right] \circ\left\langle\left[\lambda \gamma \cdot N_{1}\right], \ldots,\left[\lambda \gamma \cdot N_{m}\right]\right\rangle,\left[\lambda \alpha \cdot M_{2}\right] \circ\left\langle\left[\lambda \gamma \cdot N_{1}\right], \ldots,\left[\lambda \gamma \cdot N_{m}\right]\right\rangle\right\rangle \\
& \stackrel{*}{\rightarrow} A p p \circ\left\langle\left[\lambda \gamma \cdot M_{1}\left[x_{1}:=N_{1}, \ldots, x_{m}:=N_{m}\right]\right],\left[\lambda \gamma \cdot M_{2}\left[x_{1}:=N_{1}, \ldots, x_{m}:=N_{m}\right]\right]\right\rangle .
\end{aligned}
$$

(by induction hypothesis)

Now, when $M_{1} M_{2}\left[x_{1}:=N_{1}, \ldots, x_{m}:=N_{m}\right]$ is not of the form $c L_{1} \ldots L_{s}(c$ is an $s$-ary constant), the last term is identical with $\left[\lambda \gamma \cdot M_{1} M_{2}\left[x_{1}:=N_{1}, \ldots, x_{m}:=N_{m}\right]\right]$. Suppose that $M_{1} M_{2}\left[x_{1}:=N_{1}, \ldots, x_{m}:=N_{m}\right]$ is $c L_{1} \ldots L_{s}$. Then $M_{2}\left[x_{1}:=N_{1}, \ldots, x_{m}:=N_{m}\right]$ is $L_{\boldsymbol{s}}$. The above last term is:

$$
\begin{aligned}
& \equiv A p p \circ\left\langle\left[\lambda \gamma . c L_{1} \ldots L_{s-1}\right],\left[\lambda \gamma . L_{s}\right]\right\rangle \\
& \stackrel{*}{\rightarrow} A p p \circ\left\langle\Lambda\left(c \circ\left\langle\left[\lambda \gamma . L_{1}\right] \circ \pi_{1}^{n, 1}, \ldots,\left[\lambda \gamma . L_{s-1}\right] \circ \pi_{1}^{n, 1}, p_{n+1}^{n+1}\right\rangle\right),\left[\lambda \gamma . L_{s}\right]\right\rangle \\
& \text { (by Proposition 5.1) } \\
& \rightarrow\left(c \circ\left\langle\left[\lambda \gamma . L_{1}\right] \circ \pi_{1}^{n, 1}, \ldots,\left[\lambda \gamma . L_{s-1}\right] \circ \pi_{1}^{n, 1}, p_{n+1}^{n+1}\right\rangle\right) \circ\left\langle p_{1}^{n}, \ldots, p_{n}^{n},\left[\lambda \gamma . L_{s}\right]\right\rangle \\
& \stackrel{*}{\rightarrow} c \circ\left\langle\left[\lambda \gamma . L_{1}\right], \ldots,\left[\lambda \gamma . L_{s-1}\right],\left[\lambda \gamma . L_{s}\right]\right\rangle
\end{aligned}
$$




$$
\equiv\left[\lambda \gamma . c L_{1} \ldots L_{s-1} L_{s}\right]
$$

\section{Relationship between $C C L M_{\beta}$ and lambda calculus.}

Now we are in a position to state the theorems which describe the relationship between the terms and reductions of the two systems $C C L M_{\beta}$ and $\lambda$, in terms of the two translation algorithms of the previous section.

Theorem 6.1. Let $\gamma=\left\langle z_{1}, \ldots, z_{n}\right\rangle$. Let $M$ and $N$ be terms in $\lambda$, and the free variables in them are all in $\gamma$. If $M \stackrel{*}{\longrightarrow} \lambda N$, then $[\lambda \gamma \cdot M] \stackrel{*}{\longrightarrow}_{c}[\lambda \gamma \cdot N]$.

Proof. By induction on the definition of $M \stackrel{*}{\longrightarrow} \lambda$.

Case 1. $\alpha$-rule.

$$
\begin{aligned}
& {\left[\lambda \gamma \cdot\left(\lambda x \cdot M_{1}\right)\right]} \\
& \equiv \Lambda_{n+1}\left(\left[\lambda \gamma x^{\prime} \cdot M_{1}\left[x:=x^{\prime}\right]\right]\right) \\
& \equiv \Lambda_{n+1}\left(\left[\lambda \gamma y^{\prime} \cdot M_{1}\left[x:=y^{\prime}\right]\right]\right) \\
& \equiv\left[\lambda \gamma \cdot\left(\lambda y \cdot M_{1}[x:=y]\right)\right] .
\end{aligned}
$$

Case 2. $\beta$-rule.

$$
\begin{aligned}
& {\left[\lambda \gamma \cdot\left(\lambda x \cdot M_{1}\right) M_{2}\right]} \\
& \equiv A p p \circ\left\langle\Lambda_{n+1}\left(\left[\lambda \gamma x^{\prime} \cdot M_{1}\left[x:=x^{\prime}\right]\right]\right),\left[\lambda \gamma \cdot M_{2}\right]\right\rangle \\
& \left.\rightarrow\left[\lambda \gamma x^{\prime} \cdot M_{1}\left[x:=x^{\prime}\right]\right] \circ\left\langle p_{1}^{n}, \ldots, p_{n}^{n},\left[\lambda \gamma \cdot M_{2}\right]\right\rangle \quad \text { (by rule } 5\right) \\
& \equiv\left[\lambda \gamma x^{\prime} \cdot M_{1}\left[x:=x^{\prime}\right]\right] \circ\left\langle\left[\lambda \gamma \cdot z_{1}\right], \ldots,\left[\lambda \gamma \cdot z_{n}\right],\left[\lambda \gamma \cdot M_{2}\right]\right\rangle \\
& \stackrel{*}{\rightarrow}\left[\lambda \gamma \cdot M_{1}\left[x:=M_{2}\right]\right] . \quad \quad \text { (by Lemma }
\end{aligned}
$$

Case 3. $M \equiv \lambda x \cdot M_{1}, N \equiv \lambda x \cdot N_{1}$, and $M_{1} \stackrel{*}{\rightarrow} N_{1}$.

$\left[\lambda \gamma \cdot\left(\lambda x . M_{1}\right)\right]$

$$
\begin{aligned}
& \equiv \Lambda\left(\left[\lambda \gamma x^{\prime} \cdot M_{1}\left[x:=x^{\prime}\right]\right]\right) \\
& \stackrel{*}{\rightarrow} \Lambda\left(\left[\lambda \gamma x^{\prime} \cdot N_{1}\left[x:=x^{\prime}\right]\right]\right) \\
& \equiv\left[\lambda \gamma .\left(\lambda x . N_{1}\right)\right] .
\end{aligned}
$$

(by induction hypothesis)

Case $4 . M \equiv M_{1} M_{2}, N \equiv N_{1} N_{2}, M_{1} \stackrel{*}{\rightarrow} N_{1}$, and $M_{2} \stackrel{*}{\rightarrow} N_{2}$.

(4-1) Suppose that $M$ is of the form $c L_{1} \ldots L_{s}$, where $c$ is an $s$-ary constant. Then $N$ is also of the form $c L_{1}^{\prime} \ldots L_{s}^{\prime}$, and $L_{1} \stackrel{*}{\rightarrow} L_{1}^{\prime}, \ldots, L_{s} \stackrel{*}{\rightarrow} L_{s}^{\prime}$. Therefore, 


$$
\begin{aligned}
& {\left[\lambda \gamma \cdot M_{1} M_{2}\right]} \\
& \equiv c \circ\left\langle\left[\lambda \gamma \cdot L_{1}\right], \ldots,\left[\lambda \gamma \cdot L_{s}\right]\right\rangle \\
& \stackrel{*}{\rightarrow} c \circ\left\langle\left[\lambda \gamma \cdot L_{1}^{\prime}\right], \ldots,\left[\lambda \gamma \cdot L_{s}^{\prime}\right]\right\rangle \\
& \equiv\left[\lambda \gamma \cdot N_{1} N_{2}\right] .
\end{aligned}
$$

(by induction hypothesis)

(4-2) Suppose that $M$ is not of the form in (4-1) and that $N$ is of the form $c L_{1} \ldots L_{s}$. Then $N_{1} \equiv c L_{1} \ldots L_{s-1}$ and $N_{2} \equiv L_{s}$.

$$
\begin{aligned}
& {\left[\lambda \gamma \cdot M_{1} M_{2}\right]} \\
& \equiv A p p \circ\left\langle\left[\lambda \gamma \cdot M_{1}\right],\left[\lambda \gamma \cdot M_{2}\right]\right\rangle \\
& \stackrel{*}{\rightarrow} A p p \circ\left\langle\left[\lambda \gamma \cdot c L_{1} \ldots L_{s-1}\right],\left[\lambda \gamma \cdot L_{s}\right]\right\rangle \\
& \left.\stackrel{*}{\rightarrow}\left[\lambda \gamma \cdot c L_{1} \ldots L_{s-1} L_{s}\right]\right\rangle .
\end{aligned}
$$

(by induction hypothesis)

The last reduction has been shown in the calculation of case 5 of Lemma 5.4.

(4-3) Otherwise.

$$
\begin{aligned}
& {\left[\lambda \gamma \cdot M_{1} M_{2}\right]} \\
& \equiv A p p \circ\left\langle\left[\lambda \gamma \cdot M_{1}\right],\left[\lambda \gamma \cdot M_{2}\right]\right\rangle \\
& \stackrel{*}{\rightarrow} A p p \circ\left\langle\left[\lambda \gamma \cdot N_{1}\right],\left[\lambda \gamma \cdot N_{2}\right]\right\rangle \\
& \equiv\left[\lambda \gamma \cdot N_{1} N_{2}\right] .
\end{aligned}
$$

Theorem 6.2. Let $F$ and $G$ be in $C C L M_{\beta}$, and both are $n$-ary. If $F \stackrel{*}{\longrightarrow}_{c} G$ then

$$
F^{*}\left[N_{1}, \ldots, N_{n}\right] \stackrel{*}{\longrightarrow} G^{*}\left[N_{1}, \ldots, N_{n}\right] .
$$

Proof. By induction on the definition of $F \stackrel{*}{\rightarrow}_{c} G$.

Case 1. $F \equiv\left(H \circ\left\langle I_{1}, \ldots, I_{l}\right\rangle\right) \circ\left\langle J_{1}, \ldots, J_{m}\right\rangle$

$$
\begin{aligned}
& \quad \rightarrow G \equiv H \circ\left\langle I_{1} \circ\left\langle J_{1}, \ldots, J_{m}\right\rangle, \ldots, I_{l} \circ\left\langle J_{1}, \ldots, J_{m}\right\rangle\right\rangle . \\
& F^{*}\left[N_{1}, \ldots, N_{n}\right] \\
& \equiv H^{*}\left[I_{1}^{*}\left[J_{1}^{*}\left[N_{1}, \ldots, N_{n}\right], \ldots, J_{m}^{*}\left[N_{1}, \ldots, N_{n}\right]\right], \ldots,\right. \\
& \left.\quad I_{l}^{*}\left[J_{1}^{*}\left[N_{1}, \ldots, N_{n}\right], \ldots, J_{m}^{*}\left[N_{1}, \ldots, N_{n}\right]\right]\right] \\
& \equiv G^{*}\left[N_{1}, \ldots, N_{n}\right] .
\end{aligned}
$$

Case 2. $F \equiv p_{i}^{m} \circ\left\langle H_{1}, \ldots, H_{m}\right\rangle \rightarrow G \equiv H_{i}$.

$F^{*}\left[N_{1}, \ldots, N_{n}\right]$

$\equiv\left(p_{i}^{m}\right)^{*}\left[H_{1}^{*}\left[N_{1}, \ldots, N_{n}\right], \ldots, H_{m}^{*}\left[N_{1}, \ldots, N_{n}\right]\right]$

$\equiv G^{*}\left[N_{1}, \ldots, N_{n}\right]$.

Case 3. $F \equiv H \circ\left\langle p_{1}^{n}, \ldots, p_{n}^{n}\right\rangle \rightarrow G \equiv H$.

$$
\begin{aligned}
& F^{*}\left[N_{1}, \ldots, N_{n}\right] \\
& \equiv H^{*}\left[\left(p_{1}^{n}\right)^{*}\left[N_{1}, \ldots, N_{n}\right], \ldots,\left(p_{n}^{n}\right)^{*}\left[N_{1}, \ldots, N_{n}\right]\right] \\
& \equiv G^{*}\left[N_{1}, \ldots, N_{n}\right] .
\end{aligned}
$$

Case 4. $F \equiv \Lambda_{m+1}(H) \circ\left\langle I_{1}, \ldots, I_{m}\right\rangle \rightarrow G \equiv \Lambda_{n+1}\left(H \circ\left\langle I_{1} \circ \pi_{1}^{n, 1}, \ldots, I_{m} \circ \pi_{1}^{n, 1}, p_{n+1}^{n+1}\right\rangle\right)$. $F^{*}\left[N_{1}, \ldots, N_{n}\right]$ 


$$
\begin{aligned}
& \equiv(\Lambda(H))^{*}\left[I_{1}^{*}\left[N_{1}, \ldots, N_{n}\right], \ldots, I_{m}^{*}\left[N_{1}, \ldots, N_{n}\right]\right] \\
& \equiv \lambda x \cdot H^{*}\left[I_{1}^{*}\left[N_{1}, \ldots, N_{n}\right], \ldots, I_{m}^{*}\left[N_{1}, \ldots, N_{n}\right], x\right] \\
& \equiv G^{*}\left[N_{1}, \ldots, N_{n}\right] .
\end{aligned}
$$

Case 5. $F \equiv A p p \circ\left\langle\Lambda_{n+1}(H), I\right\rangle \rightarrow G \equiv H \circ\left\langle p_{1}^{n}, \ldots, p_{n}^{n}, I\right\rangle$.

$$
\begin{aligned}
& F^{*}\left[N_{1}, \ldots, N_{n}\right] \\
& \equiv\left(\lambda x . H^{*}\left[N_{1}, \ldots, N_{n}, x\right]\right)\left(I^{*}\left[N_{1}, \ldots, N_{n}\right]\right) \\
& \rightarrow{ }_{\lambda} H^{*}\left[N_{1}, \ldots, N_{n}, I^{*}\left[N_{1}, \ldots, N_{n}\right]\right] \quad \text { (by } \beta \text {-rule) } \\
& \equiv G^{*}\left[N_{1}, \ldots, N_{n}\right] .
\end{aligned}
$$

Case 6. $F \equiv H \circ\left\langle I_{1}, \ldots, I_{m}\right\rangle, G \equiv H^{\prime} \circ\left\langle I_{1}^{\prime}, \ldots, I_{m}^{\prime}\right\rangle, H \stackrel{*}{\rightarrow} H^{\prime}, I_{1} \stackrel{*}{\rightarrow} I_{1}^{\prime}, \ldots, I_{m} \stackrel{*}{\rightarrow} I_{m}^{\prime}$.

$$
\begin{aligned}
& F^{*}\left[N_{1}, \ldots, N_{n}\right] \\
& \equiv H^{*}\left[I_{1}^{*}\left[N_{1}, \ldots, N_{n}\right], \ldots, I_{m}^{*}\left[N_{1}, \ldots, N_{n}\right]\right]
\end{aligned}
$$$$
\stackrel{*}{\rightarrow}\left(H^{\prime}\right)^{*}\left[\left(I_{1}^{\prime}\right)^{*}\left[N_{1}, \ldots, N_{n}\right], \ldots,\left(I_{m}^{\prime}\right)^{*}\left[N_{1}, \ldots, N_{n}\right]\right] \quad \text { (by induction hypothesis) }
$$$$
\equiv G^{*}\left[N_{1}, \ldots, N_{n}\right] \text {. }
$$

Case 7. $F \equiv \Lambda(H), G \equiv \Lambda\left(H^{\prime}\right)$, and $H \stackrel{*}{\rightarrow} H^{\prime}$.

By induction hypothesis.

Before going into the next Theorem 6.3, we need the following definition.

Definition. For a term $F$ in $C C L M_{\beta}$, let $F^{+}$be the term in $C C L M_{\beta}$ obtained by replacing all $n$-ary constants $f$ (including $p_{i}^{n}$ and $A p p$ ) in $F$ by $f \circ\left\langle p_{1}^{n}, \ldots, p_{n}^{n}\right\rangle$, for all $n \geq 0$.

Theorem 6.3. Let $F$ be of arity $n$, and $\gamma \equiv\left\langle z_{1}, \ldots, z_{n}\right\rangle$. Then we have

$$
F^{+} \stackrel{*}{\longrightarrow}_{c}\left[\lambda \gamma \cdot F^{*}\left[z_{1}, \ldots, z_{n}\right]\right] \text {. }
$$

Proof. By induction on the structure of $F$.

Case 1. $F \equiv p_{i}^{n}$.

$$
\begin{aligned}
& \left(p_{i}^{n}\right)^{+} \\
& \equiv p_{i}^{n} \circ\left\langle p_{1}^{n}, \ldots, p_{n}^{n}\right\rangle \\
& \rightarrow p_{i}^{n} \\
& \equiv\left[\lambda \gamma \cdot\left(p_{i}^{n}\right)^{*}\left[z_{1}, \ldots, z_{n}\right]\right] .
\end{aligned}
$$

Case 2. $F \equiv A p p$.

$$
\begin{aligned}
& A p p^{+} \\
& \equiv A p p \circ\left\langle p_{1}^{2}, p_{2}^{2}\right\rangle \\
& \equiv\left[\lambda\left\langle z_{1}, z_{2}\right\rangle . z_{1} z_{2}\right] \\
& \equiv\left[\lambda\left\langle z_{1}, z_{2}\right\rangle . A p p^{*}\left[z_{1}, z_{2}\right]\right] .
\end{aligned}
$$

Case 3. $F \equiv f$, an $n$-ary constant other than $p_{i}^{n}$ and App.

$f^{+}$ 


$$
\begin{aligned}
& \equiv f \circ\left\langle p_{1}^{n}, \ldots, p_{n}^{n}\right\rangle \\
& \equiv f \circ\left\langle\left[\lambda \gamma . z_{1}\right], \ldots,\left[\lambda \gamma . z_{n}\right]\right\rangle \\
& \equiv\left[\lambda \gamma . f z_{1} \ldots z_{n}\right] \\
& \equiv\left[\lambda \gamma \cdot f^{*}\left[z_{1}, \ldots, z_{n}\right]\right] .
\end{aligned}
$$

Case 4. $F \equiv H \circ\left\langle I_{1}, \ldots, I_{m}\right\rangle$.

$$
\begin{aligned}
& \left(H \circ\left\langle I_{1}, \ldots, I_{m}\right\rangle\right)^{+} \\
& \equiv H^{+} \circ\left\langle I_{1}^{+}, \ldots, I_{m}^{+}\right\rangle \\
& \stackrel{*}{\rightarrow}\left[\lambda \alpha \cdot H^{*}\left[x_{1}, \ldots, x_{m}\right]\right] \circ\left\langle\left[\lambda \gamma . I_{1}^{*}\left[z_{1}, \ldots, z_{n}\right]\right], \ldots,\left[\lambda \gamma . I_{m}^{*}\left[z_{1}, \ldots, z_{n}\right]\right]\right\rangle \\
& \stackrel{*}{\rightarrow}\left[\lambda \gamma \cdot H^{*}\left[I_{1}^{*}\left[z_{1}, \ldots, z_{n}\right], \ldots, I_{m}^{*}\left[z_{1}, \ldots, z_{n}\right]\right]\right] \\
& \equiv\left[\lambda \gamma \cdot\left(H \circ\left\langle I_{1}, \ldots, I_{m}\right\rangle\right)^{*}\left[z_{1}, \ldots, z_{n}\right]\right] .
\end{aligned}
$$

Case 5. $F \equiv \Lambda(H)$.

$$
\begin{aligned}
& (\Lambda(H))^{+} \\
& \equiv \Lambda\left(H^{+}\right) \\
& \stackrel{*}{\rightarrow} \Lambda\left(\left[\lambda \gamma z \cdot H^{*}\left[z_{1}, \ldots, z_{n}, z\right]\right]\right) \\
& \equiv\left[\lambda \gamma \cdot\left(\lambda z \cdot H^{*}\left[z_{1}, \ldots, z_{n}, z\right]\right)\right] \\
& \equiv\left[\lambda \gamma \cdot(\Lambda(H))^{*}\left[z_{1}, \ldots, z_{n}\right]\right] .
\end{aligned}
$$

(by induction hypothesis)

For Theorem 6.4 we need the following definition.

Definition. For a term $M$ in $\lambda$ let $M^{\circ}$ be the term obtained from $M$ by replacing all occurrences of $s$-ary constants $c$ not appearing in the form $c M_{1} \ldots M_{s}$ by $\lambda x_{1} \ldots \lambda x_{s} . c x_{1} \ldots x_{s}$.

Let $M \equiv_{\lambda} N$ mean that $M$ and $N$ are syntactically identical except bound variables.

Theorem 6.4. Let $M$ in $\lambda, \gamma \equiv\left\langle z_{1}, \ldots, z_{n}\right\rangle$, and all the free variables in $M$ are in $\gamma$. Then we have

$$
[\lambda \gamma \cdot M]^{*}\left[z_{1}, \ldots, z_{n}\right] \equiv_{\lambda} M^{\circ}
$$

Proof. Induction by the structure of $M$.

Case 1. $M \equiv z_{i} \quad(1 \leq i \leq n)$.

$$
\begin{aligned}
& {\left[\lambda \gamma . z_{i}\right]^{*}\left[z_{1}, \ldots, z_{n}\right]} \\
& \equiv\left(p_{i}^{n}\right)^{*}\left[z_{1}, \ldots, z_{n}\right] \\
& \equiv z_{i} .
\end{aligned}
$$

Case $2 . M \equiv c \quad$ (s-ary constant).

When $s=0$ it is easy. Assume $s \geq 1$. 


$$
\begin{aligned}
& {[\lambda \gamma \cdot c]^{*}\left[z_{1}, \ldots, z_{n}\right]} \\
& \equiv\left(\Lambda^{s}\left(c \circ \pi_{2}^{n, s}\right)\right)^{*}\left[z_{1}, \ldots, z_{n}\right] \\
& \equiv \lambda x_{1} \ldots \lambda x_{s} \cdot\left(\left(c \circ \pi_{2}^{n, s}\right)^{*}\left[z_{1}, \ldots, z_{n}, x_{1}, \ldots, x_{s}\right]\right) \\
& \equiv \lambda x_{1} \ldots \lambda x_{s} . c x_{1} \ldots x_{s} .
\end{aligned}
$$

Case 3. $M \equiv \lambda x \cdot M_{1}$.

$$
\begin{aligned}
& {\left[\lambda \gamma \cdot\left(\lambda x \cdot M_{1}\right)\right]^{*}\left[z_{1}, \ldots, z_{n}\right]} \\
& \equiv\left(\Lambda_{n+1}\left(\left[\lambda \gamma x^{\prime} \cdot M_{1}\left[x:=x^{\prime}\right]\right]\right)\right)^{*}\left[z_{1}, \ldots, z_{n}\right] \\
& \equiv \lambda y \cdot\left(\left[\lambda \gamma x^{\prime} \cdot M_{1}\left[x:=x^{\prime}\right]\right]^{*}\left[z_{1}, \ldots, z_{n}, y\right]\right) \\
& \equiv_{\lambda} \lambda y \cdot M_{1}^{\circ}[x:=y] .
\end{aligned}
$$

Case 4. $M \equiv c M_{1} \ldots M_{s} \quad(c$ is an $s$-ary constant $)$.

$$
\begin{aligned}
& {\left[\lambda \gamma . c M_{1} \ldots M_{s}\right]^{*}\left[z_{1}, \ldots, z_{n}\right]} \\
& \equiv\left(c \circ\left\langle\left[\lambda \gamma \cdot M_{1}\right], \ldots,\left[\lambda \gamma \cdot M_{s}\right]\right\rangle\right)^{*}\left[z_{1}, \ldots, z_{n}\right] \\
& \equiv c\left(\left[\lambda \gamma \cdot M_{1}\right]^{*}\left[z_{1}, \ldots, z_{n}\right]\right) \ldots\left(\left[\lambda \gamma \cdot M_{s}\right]^{*}\left[z_{1}, \ldots, z_{n}\right]\right) \\
& \equiv_{\lambda} c M_{1}^{\circ} \ldots M_{s}^{\circ} . \quad \text { (by induction hypothesis) }
\end{aligned}
$$

Case 5. $M \equiv M_{1} M_{2}$, and not of the form of case 4 .

$\left[\lambda \gamma \cdot M_{1} M_{2}\right]^{*}\left[z_{1}, \ldots, z_{n}\right]$

$$
\begin{aligned}
& \equiv\left(A p p \circ\left\langle\left[\lambda \gamma \cdot M_{1}\right],\left[\lambda \gamma \cdot M_{2}\right]\right)\right)^{*}\left[z_{1}, \ldots, z_{n}\right] \\
& \equiv\left(\left[\lambda \gamma \cdot M_{1}\right]^{*}\left[z_{1}, \ldots, z_{n}\right]\right)\left(\left[\lambda \gamma \cdot M_{2}\right]^{*}\left[z_{1}, \ldots, z_{n}\right]\right) \\
& \equiv_{\lambda} M_{1}^{\circ} M_{2}^{\circ} .
\end{aligned}
$$

(by induction hypothesis) 


\section{Church-Rosser property.}

In this section we prove the confluency property for $C C L M_{\beta}$.

Definition. For a term $F$ in $C C L M_{\beta}$ define the term $F^{-}$in $C C L M_{\beta}$ as follows.

1. For a constant $f$, including $p_{i}^{n}$ and $A p p, f^{-} \equiv f$.

2. $\left(G \circ\left\langle H_{1}, \ldots, H_{n}\right\rangle\right)^{-} \equiv \begin{cases}H_{i}^{-} & \text {if } G^{-} \equiv p_{i}^{n}, \\ G^{-} & \text {if }\left\langle H_{1}^{-}, \ldots, H_{n}^{-}\right\rangle \equiv\left\langle p_{1}^{n}, \ldots, p_{n}^{n}\right\rangle, \\ G^{-} \circ\left\langle H_{1}^{-}, \ldots, H_{n}^{-}\right\rangle & \text {otherwise. }\end{cases}$

3. $(\Lambda(G))^{-} \equiv \Lambda\left(G^{-}\right)$.

Thus, $F^{-}$is the term obtained from $F$ by applying reductions concerning projections (rules 2 and 3 ) as much as possible. We state without proof easy properties of this transformation.

\section{Lemma 7.1.}

i) $\quad F \stackrel{*}{\longrightarrow} F^{-}$.

ii) $\quad\left(F^{-}\right)^{-} \equiv F^{-}$.

iii) $\quad F \stackrel{*}{\longrightarrow}\left(F^{+}\right)^{-}$.

Lemma 7.2. $F \stackrel{*}{\longrightarrow}$ c $G$ then $F^{-} \stackrel{*}{\longrightarrow}_{c} G^{-}$.

Proof. By induction on the length of reduction of $F \stackrel{*}{\rightarrow} G$.

Case 1. $F \equiv\left(H \circ\left\langle I_{1}, \ldots, I_{m}\right\rangle\right) \circ\left\langle J_{1}, \ldots, J_{n}\right\rangle \rightarrow G \equiv H \circ\left\langle I_{1} \circ\left\langle J_{1}, \ldots, J_{n}\right\rangle, \ldots\right\rangle$.

(1-1) $H^{-} \equiv p_{i}^{m}$.

$$
F^{-} \equiv\left(I_{i} \circ\left\langle J_{1}, \ldots, J_{n}\right\rangle\right)^{-} \equiv G^{-} .
$$

(1-2) $\left\langle I_{1}^{-}, \ldots, I_{m}^{-}\right\rangle \equiv\left\langle p_{1}^{m}, \ldots, p_{m}^{m}\right\rangle \quad$ (necessarily $m=n$ ).

$$
F^{-} \equiv\left(H \circ\left\langle J_{1}, \ldots, J_{n}\right\rangle\right)^{-} \equiv G^{-} \text {. }
$$

$(1-3)\left\langle J_{1}^{-}, \ldots, J_{n}^{-}\right\rangle \equiv\left\langle p_{1}^{n}, \ldots, p_{n}^{n}\right\rangle$.

$$
F^{-} \equiv\left(H \circ\left\langle I_{1}, \ldots, I_{m}\right\rangle\right)^{-} \equiv G^{-} .
$$

(1-4) Otherwise.

$$
F^{-} \equiv\left(H^{-} \circ\left\langle I_{1}^{-}, \ldots, I_{m}^{-}\right\rangle\right) \circ\left\langle J_{1}^{-}, \ldots, J_{n}^{-}\right\rangle \rightarrow H^{-} \circ\left\langle I_{1}^{-} \circ\left\langle J_{1}^{-}, \ldots, J_{n}^{-}\right\rangle, \ldots\right\rangle \equiv G^{-} .
$$

Case 2. $F \equiv p_{i}^{n} \circ\left\langle H_{1}, \ldots, H_{n}\right\rangle \rightarrow G \equiv H_{i}$.

$$
F^{-} \equiv H_{i}^{-} \equiv G^{-} \text {. }
$$

Case 3. $F \equiv H \circ\left\langle p_{1}^{n}, \ldots, p_{n}^{n}\right\rangle \rightarrow G \equiv H$.

$$
F^{-} \equiv H^{-} \equiv G^{-} \text {. }
$$

Case 4. $F \equiv \Lambda(H) \circ\left\langle I_{1}, \ldots, I_{n}\right\rangle^{k} \rightarrow G \equiv \Lambda\left(H \circ\left\langle I_{1} \circ \pi_{1}^{k, 1}, \ldots, I_{n} \circ \pi_{1}^{k, 1}, p_{k+1}^{k+1}\right\rangle\right)$.

(4-1) $\left\langle I_{1}^{-}, \ldots, I_{n}^{-}\right\rangle \equiv\left\langle p_{1}^{n}, \ldots, p_{n}^{n}\right\rangle$.

$$
F^{-} \equiv \Lambda\left(H^{-}\right) \equiv G^{-} \text {. }
$$


(4-2) $H^{-} \equiv p_{i}^{n+1}$, and not of the form of (4-1).

$$
\begin{aligned}
& F^{-} \equiv \Lambda\left(H^{-}\right) \circ\left\langle I_{1}^{-}, \ldots, I_{n}^{-}\right\rangle \\
& \rightarrow \Lambda\left(p_{i}^{n+1} \circ\left\langle I_{1}^{-} \circ \pi_{1}^{k, 1}, \ldots, I_{n}^{-} \circ \pi_{1}^{k, 1}, p_{k+1}^{k+1}\right\rangle\right) \\
& \rightarrow G^{-} .
\end{aligned}
$$

(4-3) Otherwise.

$$
\begin{aligned}
& F^{-} \equiv \Lambda\left(H^{-}\right) \circ\left\langle I_{1}^{-}, \ldots, I_{n}^{-}\right\rangle \\
& \rightarrow \Lambda\left(H^{-} \circ\left\langle I_{1}^{-} \circ \pi_{1}^{k, 1}, \ldots, I_{n}^{-} \circ \pi_{1}^{k, 1}, p_{k+1}^{k+1}\right\rangle\right) \\
& \equiv G^{-} .
\end{aligned}
$$

Case 5. $F \equiv A p p \circ\langle\Lambda(H), I\rangle \rightarrow G \equiv H \circ\left\langle p_{1}^{n}, \ldots, p_{n}^{n}, I\right\rangle$.

(5-1) $H^{-} \equiv p_{i}^{n+1}$.

$$
\begin{aligned}
& F^{-} \equiv A p p \circ\left\langle\Lambda\left(H^{-}\right), I^{-}\right\rangle \\
& \rightarrow p_{i}^{n+1} \circ\left\langle p_{1}^{n}, \ldots, p_{n}^{n}, I^{-}\right\rangle \\
& \rightarrow G^{-} .
\end{aligned}
$$

(5-2) Otherwise.

$$
\begin{aligned}
& F^{-} \equiv A p p \circ\left\langle\Lambda\left(H^{-}\right), I^{-}\right\rangle \\
& \rightarrow H^{-} \circ\left\langle p_{1}^{n}, \ldots, p_{n}^{n}, I^{-}\right\rangle \\
& \equiv G^{-} .
\end{aligned}
$$

Case 6. $F \equiv H \circ\left\langle I_{1}, \ldots, I_{n}\right\rangle \stackrel{*}{\rightarrow} G \equiv H^{\prime} \circ\left\langle I_{1}^{\prime}, \ldots, I_{n}^{\prime}\right\rangle, H \stackrel{*}{\rightarrow} H^{\prime}$, and $I_{1} \stackrel{*}{\rightarrow} I_{1}^{\prime}, \ldots, I_{n} \stackrel{*}{\rightarrow} I_{n}^{\prime}$. (6-1) $H \equiv p_{i}^{n}$.

Since $H^{\prime} \equiv p_{i}^{n}$, by induction hypothesis we have

$$
F^{-} \equiv I_{i}^{-} \stackrel{*}{\longrightarrow}\left(I_{i}^{\prime}\right)^{-} \equiv G^{-} .
$$

(6-2) $\left\langle I_{1}, \ldots, I_{n}\right\rangle \equiv\left\langle p_{1}^{n}, \ldots, p_{n}^{n}\right\rangle$.

Since $\left\langle I_{1}^{\prime}, \ldots, I_{n}^{\prime}\right\rangle \equiv\left\langle p_{1}^{n}, \ldots, p_{n}^{n}\right\rangle$, by induction hypothesis we have

$$
F^{-} \equiv H^{-} \stackrel{*}{\longrightarrow}\left(H^{\prime}\right)^{-} \equiv G^{-} \text {. }
$$

(6-3) Otherwise.

$$
\begin{aligned}
& F^{-} \equiv H^{-} \circ\left\langle I_{1}^{-}, \ldots, I_{n}^{-}\right\rangle \\
& \stackrel{*}{\rightarrow}\left(H^{\prime}\right)^{-} \circ\left\langle\left(I_{1}^{\prime}\right)^{-}, \ldots,\left(I_{n}^{\prime}\right)^{-}\right\rangle \quad \text { (by induction hypothesis) } \\
& \stackrel{*}{\rightarrow} G^{-} .
\end{aligned}
$$

Case 7. $F \equiv \Lambda(H) \stackrel{*}{\rightarrow} G \equiv \Lambda\left(H^{\prime}\right)$ and $H \stackrel{*}{\rightarrow} H^{\prime}$.

By induction hypothesis.

The following theorem establishes the Church-Rosser property of $C C L M_{\beta}$.

Theorem 7.3. If $F \stackrel{*}{\longrightarrow} G_{1}$, and $F \stackrel{*^{\prime}}{\longrightarrow} G_{2}$, then there exists $H$ such that $G_{1} \stackrel{*}{\longrightarrow} H$ and $G_{2} \stackrel{*}{\longrightarrow}$ $H$.

Proof. Suppose 


$$
F \stackrel{*}{\longrightarrow} G_{1} \text {, and } F \stackrel{*}{\longrightarrow}{ }_{c} G_{2} .
$$

Assume that $F$ is $n$-ary, so that $G_{1}$ and $G_{2}$ are also $n$-ary. By Theorem 6.2 we have $F^{*}\left[z_{1}, \ldots, z_{n}\right] \stackrel{*}{\longrightarrow} \lambda G_{1}^{*}\left[z_{1}, \ldots, z_{n}\right]$, and $F^{*}\left[z_{1}, \ldots, z_{n}\right] \stackrel{*}{\longrightarrow} \lambda G_{2}^{*}\left[z_{1}, \ldots, z_{n}\right]$.

By the Church-Rosser theorem of the type-free $\lambda_{\beta}$-calculus, there exists $M$ such that

$$
G_{1}^{*}\left[z_{1}, \ldots, z_{n}\right] \stackrel{*}{\longrightarrow} \lambda M \text {, and } G_{2}^{*}\left[z_{1}, \ldots, z_{n}\right] \stackrel{*}{\longrightarrow} M .
$$

Let $\gamma \equiv\left\langle z_{1}, \ldots, z_{n}\right\rangle$. By Theorem 6.1 we have

$$
\left[\lambda \gamma \cdot G_{1}^{*}\left[z_{1}, \ldots, z_{n}\right]\right] \stackrel{*}{\longrightarrow}_{c}[\lambda \gamma \cdot M] \text {, and }\left[\lambda \gamma \cdot G_{2}^{*}\left[z_{1}, \ldots, z_{n}\right]\right] \stackrel{*}{\longrightarrow}_{c}[\lambda \gamma \cdot M] .
$$

By the way, by Theorem 6.3 we have

$$
G_{1}^{+} \stackrel{*}{\longrightarrow}_{c}\left[\lambda \gamma \cdot G_{1}^{*}\left[z_{1}, \ldots, z_{n}\right]\right] \text {, and } G_{2}^{+} \stackrel{*}{\longrightarrow}_{c}\left[\lambda \gamma \cdot G_{2}^{*}\left[z_{1}, \ldots, z_{n}\right]\right] .
$$

By combining (1) and (2) we get

$$
G_{1}^{+} \stackrel{*}{\longrightarrow}_{c}[\lambda \gamma \cdot M] \text {, and } G_{2}^{+} \stackrel{*}{\longrightarrow}_{c}[\lambda \gamma \cdot M] .
$$

Applying Lemma 7.2 to the above we have

$$
\left(G_{1}^{+}\right)^{-} \stackrel{*}{\longrightarrow}_{c}[\lambda \gamma \cdot M]^{-}, \text {and }\left(G_{2}^{+}\right)^{-} \stackrel{*}{\longrightarrow}_{c}[\lambda \gamma \cdot M]^{-} .
$$

Therefore, if we put $H$ as $[\lambda \gamma \cdot M]^{-}$, by using Lemma $\left.7.1 \mathrm{iii}\right)$ we have

$$
G_{1} \stackrel{*}{\longrightarrow}_{c}\left(G_{1}^{+}\right)^{-} \stackrel{*}{\longrightarrow} c H \text {, and } G_{2} \stackrel{*}{\longrightarrow}_{c}\left(G_{2}^{+}\right)^{-} \stackrel{*}{\longrightarrow}_{c} H \text {. }
$$

The proof is completed.

Remark. We have reduced the proof of Church-Rosser property of $C C L M_{\beta}$ to that of lambda calculus by using the relationship of the reductions between the two systems. A direct proof, that is, a proof solely within the system, may be possible, but it will perhaps be a tedious one. For the $C C M$ calculus of Yokouchi, a direct proof of the property is in [14]. 


\section{REFERENCES}

[1] J. Backus, Can programming be liberated from the von Neumann style? A functional style and its algebra of programs, Comm. ACM, 21 (1978), pp. 613-641.

[2] H. P. Barendregt, The Lambda Calculus: Its Syntax and Semantics, Revised ed., NorthHolland, Amsterdam, 1984.

[3] G. Cousineau, P.-L. Curien and M. Mauny, The categorical abstract machine, Sci. Comput. Program., 8 (1987), pp. 173-202.

[4] P.-L. Curien, Categorical combinators, Inform. Contr., 69 (1986), pp. 188-254.

[5] P.-L. Curien, Categorical Combinators, Sequential Algorithms and Functional Programming, Pitman, London, 1986.

[6] Y. Futamura, Partial computation of programs, Lecture Notes in Computer Science, vol. 147, pp. 1-35, Springer-Verlag, Berlin, 1983.

[7] S. Hayashi, Adjunction of semifunctors: Categorical structures in nonextensional lambda calculus, Theoret. Comput. Sci., 41 (1985), pp. 95-104.

[8] C. P. J. Koymans, Models of the lambda calculus, Inform. Contr., 52 (1982), pp. 306-332.

[9] J. Lambek, Functional completeness of cartesian categories, Ann. Math. Logic, 6 (1974), pp. 259-292.

[10] J. Lambek and P. J. Scott, Introduction to Higher Order Categorical Logic, Cambridge University Press, Cambridge, 1986.

[11] A. R. Meyer, What is a model of the lambda calculus?, Inform. Contr., 52 (1982), pp. $87-122$.

[12] H. Yokouchi, Application and composition in functional programming, J. Inform. Process., 8 (1985), pp. 217-221.

[13] H. Yokouchi, Retraction map categories and their applications to the construction of lambda calculus models, Inform. Contr., 71 (1986), pp. 33-86.

[14] H. Yokouchi, The CCM calculus - another formalization of the $\lambda$-calculus, preprint, 1987. 